\title{
OBSERVATIONS OF SHOCKED REGIONS
}

\author{
L. M. Ziurys \\ Department of Chemistry \\ Arizona State University \\ Tempe, AZ 85287-1604
}

ABSTRACT. Recent observations of molecular clouds perturbed by interstellar shocks are
reviewed. Effects of the shock waves on the chemical composition of these regions are discussed.

\section{Introduction}

Interstellar shocks occur when the bulk fluid velocity of a gas exceeds the local sound speed. Shocks heat, compress, and accelerate the gas, changing its physical and chemical characteristics substantially. Since the local sound speed in a molecular cloud is usually $\approx 0.3 \mathrm{~km} / \mathrm{s}$, shocks should be common phenomena.

There are various types of shocks, including fast, dissociate "J-type" ones. These satisfy the so-called "jump" conditions, and generally destroy molecules. Lower velocity C-type shocks are thought to involve magnetic precursors (e.g., McKee, Chemhoff, Hollenbach 1983). In the latter type, molecules aren't dissociated, and thus shocked regions studied by molecular-line radio astronomy are thought to be of this kind.

The passage of a shock wave through cold, unperturbed gas is thought to affect its chemical composition. Not only can dust grains be destroyed, releasing "frozen-out" species, but the rise in temperature and pressure can also significantly change ambient gas phase abundances. At low temperatures typical of cold, quiescent molecular clouds, i.e., $\mathrm{T} \sim 10 \mathrm{~K}$, endothermic reactions, and ones with any significant activation barrier, cannot occur on a reasonable time scale. A rise in temperature, anywhere from 100 to thousands of degrees as a result of a shock wave, can open these new chemical channels and critically alter expected "ion-molecule"-type abundances.

Many "shock chemistry" models have been made to examine the chemistry behind a shock wave (e.g., Hartquist, Oppenheimer and Dalgarno 1980; Mitchell 1984; Neufeld and Dalgarno 1989). Although the details of individual models differ, there are some common conclusions: $\mathrm{CO}$ is thought to be unaffected chemically by a shock, while abundances of certain oxygen-containing molecules such as $\mathrm{OH}, \mathrm{H}_{2} \mathrm{O}$, and $\mathrm{SiO}$, and certain sulfur species $\left(\mathrm{H}_{2} \mathrm{~S}, \mathrm{SO}\right.$, and $\left.\mathrm{SO}_{2}\right)$ are enhanced due to the occurrence of reactions with energy barriers.

Observationally, shocked regions are discovered by the presence of unusually broad, often asymmetric line profiles found in molecular spectra. Line profiles from quiescent gas are generally quite narrow. A classic example of a molecular line which is thought to show the presence of a 
shock wave is the $\mathrm{J}=1 \rightarrow 0$ line of $\mathrm{CO}$, observed toward the B cloud near the supernova remnant IC443 (DeNoyer and Frerking 1981). A narrow "spike"-type feature is found near $V_{L S R} \approx+5$ $\mathrm{km} / \mathrm{s}$, and is thought to represent the cold, unperturbed "pre-shock" gas. Nearby in velocity is a broad, asymmetric line that extends from $V_{L S R}=-5$ to $-60 \mathrm{~km} / \mathrm{s}$, thought to trace material that has been hit by a shock wave from the supernova explosion. This gas presumably has been heated and accelerated, hence the abrupt change in LSR velocity, as well as the presence of emission at velocities far larger than that of the quiescent gas.

Besides broad line profiles, there are other possible tracers of shock waves. These include the presence of vibrationally excited $\mathrm{H}_{2}$ emission, as well as the appearance of highly-excited lines of molecules such as $\mathrm{CO}, \mathrm{HCN}$, and $\mathrm{OH}$, particularly in the far IR. However, there may be other mechanisms that could be exciting these tracers, such as imbedded infrared sources.

Because shock waves are readily generated when stars form, as well as when they destruct, observations of shocked regions should be in abundance. However, only a few such objects have been looked at in great detail. These include the Orion-KL high mass star-forming region, a few cloud complexes which have been subjected to a supernovae blast (IC443, W28, W44), and a few loss-mass star-forming objects, which include L1551 and Barnard 1.

\section{Shocked Gas in a Region of High Mass Star-Formation: Orion-KL}

The shocked gas in Orion is associated with various outflows present in the region. A young $\sim 25 \mathrm{M} \Theta$ star, presumably IRc2, has formed in the quiescent cloud OMC-1. In the course of its evolution, it has undergone mass loss, initiating several outflows that shock and heat the surrounding gas (e.g., Plambeck et al. 1985). Several kinematic components and chemical components exist in this region, introducing a rather complicated morphology. There is the ambient, quiescent material of OMC-1, which is called the "spike" or "ridge." Then there are the outflow regions, which are centered near IRc2. There is thought to be high velocity, "extended" bipolar outflow, and a lower velocity one, sometimes called the "doughnut" or "plateau." These outflows probably represent shocked gas since they are rather hot $\left(\mathrm{T}_{\mathbf{k}} \sim 100 \mathrm{~K}\right)$, and dense, the high velocity plateau exhibiting $n\left(\mathrm{H}_{2}\right) \approx 10^{5} \mathrm{~cm}^{-3}$, and the low velocity one having $\mathrm{n}\left(\mathrm{H}_{2}\right) \geq 10^{6}$ $\mathrm{cm}^{-3}$.

Another region of interest in Orion-KL is the "hot core," a dense clump or clumps of material a few arcseconds in distance from IRc2, with $\mathrm{T}_{\mathrm{k}}-200 \mathrm{~K}$ and $\mathrm{N}\left(\mathrm{H}_{2}\right) \sim 10^{7} \mathrm{~cm}^{-3}$ (e.g. Plambeck et al.1985). This region of hot dense gas may or may not actually be heated as a result of shock waves. In the initial stages of the mass loss from IRc2, the hot core may have been subjected to a shock wave blast, which elevated its temperature and density (Masson et al. 1985). This is supported by the presence of a so-called cavity around IRc2 (Wynn-Williams et al. 1984). Alternately, the hot core may be gas that is primarily heated by IRc2, or a highly imbedded, young star (Plambeck et al. 1985).

There have been numerous studies of the shocked regions in Orion, including several spectral line surveys carried out at mm-wavelengths (e.g., Johansson et al. 1984; Sutton et al. 1985; Jewell et al. 1989; Ziurys and McGonagle 1991). Such surveys are useful because the hot core and plateau regions, i.e. the shocked material in Orion, are usually distinguishable from the quiescent gas in a single line profile. Thus, it is possible to assign a species as arising from shocked or quiescent gas, or both.

The molecules that appear to be quite abundant in the "plateau" outflows are $\mathrm{SO}, \mathrm{SO}_{2}, \mathrm{H}_{2} \mathrm{~S}$, SiO, and SiS. (e.g. Johansson et al. 1984; Blake et al. 1987; Minh et al. 1990). Sulfur and 
silicon molecules do appear to be preferentially produced in shocked gas, at least in Orion. SO, $\mathrm{SO}_{2}$, and $\mathrm{H}_{2} \mathrm{~S}$ are abundant in the hot core, as well, but have much reduced concentrations in the ridge (e.g., Minh et al. 1990; Ziurys and McGonagle 1991). In contrast , silicon species do not usually appear in the quiescent gas (Ziurys, Friberg, Irvine 1989).

There are many species present in the hot core, including $\mathrm{NH}_{3}, \mathrm{HCN}$, etc., that are also abundant in the ridge as well. Ethyl cyanide, however, appears to be only in the hot core (e.g. Johansson et al. 1984; Sutton et al. 1985; Ziurys and McGonagle1991). It, therefore, may be exclusively produced by shocks. Blake et al. (1987) attribute the behavior of EtCN to grain processing, which hydrogenates double and triple carbon bonds. Yet, $\mathrm{HC}_{3} \mathrm{~N}$, which contains carbon-carbon triple bond, is prominent in the hot core as well.

The dominant presence of EtCN in the hot core is curious. In contrast, other large organic species such as dimethyl ether $\left(\left(\mathrm{CH}_{3}\right)_{2} \mathrm{O}\right)$ and methyl acetylene $\left(\mathrm{H}_{3} \mathrm{C}-\mathrm{C} \equiv \mathrm{CH}\right)$, are exclusively observed in the ridge (e.g., Ziurys, Wilson, and Waterlout 1991). The chemistry of large organic compounds in interstellar gas is thus very selective, and has yet to be satisfactorily explained.

\section{Shocked Gas Near Supernova Remnants}

Supernova remnants which demonstrate a clear-cut interaction of molecular cloud and blast wave are relatively rare (e.g., Wootten, 1978). One case where there does appear to be a definite interaction is the molecular cloud complex associated with the young SNR IC443. There may also be some interaction in SNR W28, and perhaps in W44, but the evidence is less convincing.

Shocked gas was initially detected in IC443 through the observation of molecular line profiles with extremely large velocity dispersions in several nearby clouds (e.g., DeNoyer 1979; DeNoyer and Frerking 1981). These spectra were measured at several positions near the SNR, identifying small, perturbed regions of gas referred to as clouds or "clumps" A, B, and C. Broad line profiles were observed in $\mathrm{CO}, \mathrm{HCO}^{+}, \mathrm{HCN}, \mathrm{H}_{2} \mathrm{CO}, \mathrm{OH}$, and $\mathrm{CS}$.

More recent studies by Dickman et al. (1991) reveal an even more interesting structure. Large scale mapping of the $\mathrm{J}=1 \rightarrow 0$ transition of $\mathrm{HCO}^{+}$shows the presence of at least six more highly perturbed clumps. Together with clouds A, B, and C, they form a roughly elliptical ring about 9 pc across on the face of the optical SNR. This is illustrated in Figure 1. The bulk of the redshifted emission is observed on the northern section of the ellipse and the blue-shifted emission on the southern part, which suggest the clumps lie along the edge of a tilting, expanding ring.

Of the nine clumps associated with IC443, only two have been examined in a variety of molecules. These are the most perturbed regions, clumps B and G. Ziurys, Snell and Dickman (1989) studied the previously-detected species $\left(\mathrm{HCN}, \mathrm{HCO}^{+}, \mathrm{CO}\right.$, and CS), and observed several new molecules towards these regions, including $\mathrm{SiO}, \mathrm{SO}, \mathrm{N}_{2} \mathrm{H}^{+}, \mathrm{HNC}, \mathrm{CN}$, and $\mathrm{NH}_{3}$. They also detected the $\mathrm{J}=3 \rightarrow 2$ transition of $\mathrm{HCO}^{+}$in both clumps. The multi-transition study of $\mathrm{HCO}^{+}$showed that the densities in both clumps was at least $3 \times 10^{5} \mathrm{~cm}^{-3}$. Analysis of the $(1,1)$ and $(2,2)$ inversion transitions of $\mathrm{NH}_{3}$ showed that the gas kinetic temperature was $\mathrm{T}_{k} \geq 40 \mathrm{~K}$. These densities and temperatures strongly indicate shock heating and compression. 


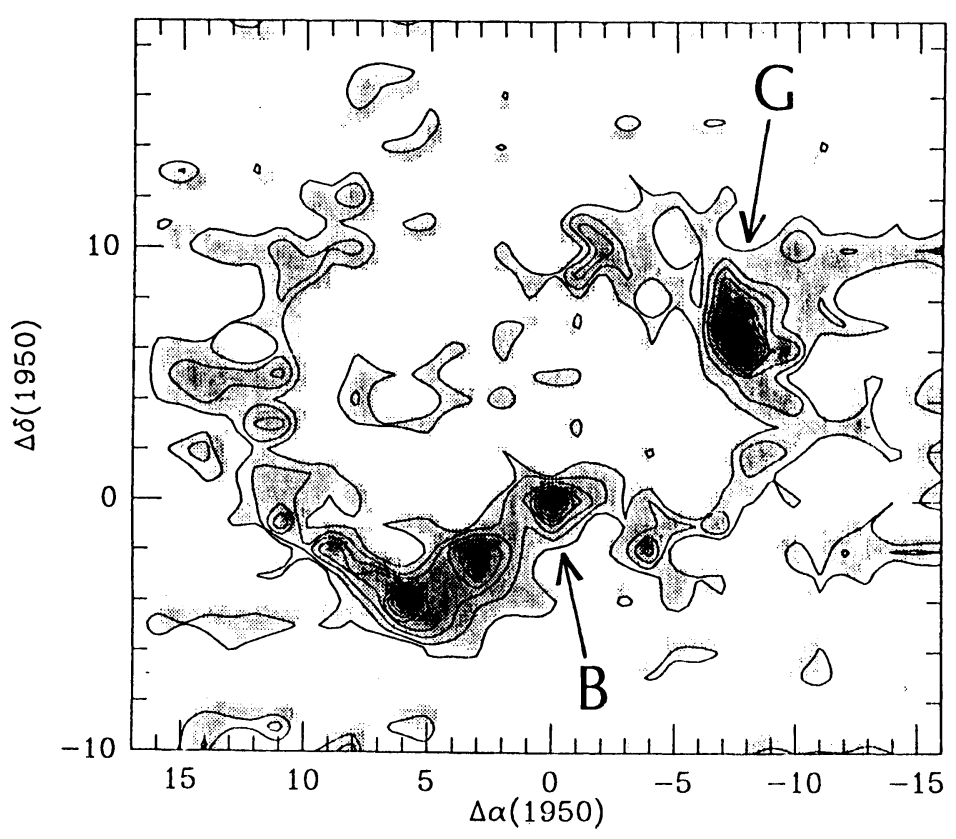

Figure 1. Contours of integrated $\mathrm{J}=1 \rightarrow 0 \mathrm{HCO}^{+}$emission, showing a "ring" of perturbed clumps in SNR IC443. The $(0,0)$ position is $\alpha=06^{\mathrm{h}} 14^{\mathrm{m}} 15^{\mathrm{s}} ; \delta=22^{\circ} 26^{\prime} 50^{\prime \prime}$ (1950.0). Positions of clumps Band $G$ are indicated (from Dickman et al. 1991).

The chemical abundances in clumps B and $\mathrm{G}$ are thus likely to be affected by shock waves. A summary of chemical abundances measured toward these two regions is given in Table 1. For comparison, abundances established toward TMC-1 are also shown. Because of the large dipole moments of the species studied, relative to that of CO (1-4D vs. 0.1D), all abundances are referenced to HCN $(\mu=2.98 \mathrm{D})$. As the table shows, the only molecule whose abundance is significantly enhanced in the shocked gas of IC443 is SiO, whose concentration is 100 times higher than the upper limit found for TMC-1. In contrast, the SO abundances in IC443 are comparable to those of TMC-1, as well as for most of the other species studied. There appears to be a slight enhancement in the $\mathrm{HCO}^{+}$abundance, as well. $\mathrm{HNC}$, however, is an order of magnitude less abundant in IC443 vs. TMC-1. This is not unexpected, as HNC is a metastable isomer. Elevated temperatures allow it to convert to its more stable form, $\mathrm{HCN}$.

Broadened line profiles in molecules have also been observed towards the supernova remnants W28 and W44 (Wootten 1981; DeNoyer 1983). Towards W44, however, the moderately broadened profiles $\left(\Delta \mathrm{V}_{1 / 2} \approx 10 \mathrm{~km} / \mathrm{s}\right)$ can be explained by the presence of multiple velocity components along the line of sight, rather than resulting from shock acceleration. Towards W28, the line profiles in species such as $\mathrm{HCO}^{+}$exhibit $\Delta \mathrm{V}_{1 / 2} \approx 20 \mathrm{~km} / \mathrm{s}$, which perhaps are due to the passage of a shock. Again, $\mathrm{CO}$ shows at three individual velocity components present over the same broad velocity interval as the $\mathrm{HCO}^{+}$emission. The evidence for the presence of shocked material is therefore uncertain in this object. 
Table I Abundances in shocked regions relative to TMC-1

\begin{tabular}{|c|c|c|c|c|c|c|}
\hline Molecule & & $\begin{array}{l}\text { X/HCNa) } \\
\text { IC443 }\end{array}$ & $\begin{array}{l}\text { X/HCNa) } \\
\text { Orion } \\
\text { Plateau }\end{array}$ & $\begin{array}{l}\left.\mathrm{X} / \mathrm{HCN}^{\mathrm{b}}\right) \\
\mathrm{L} 1551 \mathrm{E}\end{array}$ & $\begin{array}{l}\left.\mathrm{X} / \mathrm{HCN}^{\mathrm{b}}\right) \\
\mathrm{L} 1551 \mathrm{~W}\end{array}$ & $\begin{array}{l}\left.\mathrm{X} / \mathrm{HCN}^{\mathrm{a}}\right) \\
\mathrm{TMC}-1\end{array}$ \\
\hline \multirow[t]{2}{*}{$\mathrm{HCO}^{+}$} & B & $1.2-1.5$ & - & 1.1 & 0.65 & 0.4 \\
\hline & G & $0.80-1.3$ & - & - & - & - \\
\hline \multirow[t]{2}{*}{$\mathrm{N}_{2} \mathrm{H}^{+}$} & B & 0.035 & - & - & - & 0.025 \\
\hline & G & 0.013 & - & - & - & - \\
\hline \multirow[t]{2}{*}{ CS } & B & 0.8 & 0.08 & 3.4 & 7.7 & 0.5 \\
\hline & G & 0.7 & - & - & - & - \\
\hline \multirow[t]{2}{*}{ SO } & B & 0.82 & 1.87 & $<0.45$ & 7.7 & 0.25 \\
\hline & G & 0.55 & - & - & - & - \\
\hline \multirow[t]{2}{*}{ SiO } & B & 0.038 & 0.1 & $<0.07$ & $<0.22$ & $<0.0005$ \\
\hline & G & 0.063 & - & - & - & - \\
\hline \multirow[t]{2}{*}{$\mathrm{HCN}$} & B & 1 & 1 & 1 & 1 & 1 \\
\hline & G & 1 & - & - & - & - \\
\hline \multirow[t]{2}{*}{ HNC } & B & 0.14 & 0.005 & - & - & 1 \\
\hline & G & 0.11 & - & - & - & - \\
\hline \multirow[t]{2}{*}{$\mathrm{CN}$} & B & 1.7 & - & - & - & 1.5 \\
\hline & G & 0.89 & - & - & - & - \\
\hline \multirow[t]{2}{*}{$\mathrm{NH}_{3}$} & B & 1.3 & 0.5 & 2.6 & 4.6 & 1 \\
\hline & G & 0.4 & - & - & - & - \\
\hline \multirow[t]{2}{*}{$\mathrm{CO}$} & B & 20,000 & 430 & - & - & 4,000 \\
\hline & G & 7,500 & - & - & - & - \\
\hline
\end{tabular}

a) From Ziurys et al. (1989); Ziurys (1990)

b) From Plambeck and Snell (1991)

\section{Shocked Gas in Regions of Low Mass Star Formation}

There are several objects where low-mass star-formation is occurring, and an associated outflow has affected nearby ambient gas. Two such sources where the chemical abundances has been investigated in detail are L1551 (Plambeck and Snell 1991) and Barnard 1 (Bachiller, Menten, and del Río-Alvarez 1990).

The outflow associated with L1551 was initially detected in broad CO line profiles (e.g. Snell, Loren and Plambeck 1980). Subsequent studies have shown that this outflow is bipolar, and is centered on the infrared source IRS-5, presumably a young star in its mass loss stage. More recent observations suggest that a complete shell of accelerated molecular material surrounds IRS-5 (Schloerb and Snell 1985). The bipolar lobes of the outflow are regions where the shell has impacted on surrounding quiescent clumps. Hence, the two lobes associated with the L1551 outflow are likely to be regions that have been shocked.

Recently, Plambeck and Snell (1991) have measured the abundances of various molecules in the L1551 bipolar lobes, L1551 E and L155W. As Table I illustrates, several of the species of interest in shocked gas were not detected. These include $\mathrm{SiO}, \mathrm{SO}$ (in L1551E), and $\mathrm{SO}_{2}$. Of the molecules detected, $\mathrm{HC}_{3} \mathrm{~N}$ and $\mathrm{NH}_{3}$ have concentrations comparable to TMC-1. The abundance of $\mathrm{HCO}^{+}$may be slightly enhanced, however, as was found for IC443. CS, on the other hand, is about an order of magnitude more abundant in both lobes of L1551 vs. TMC-1. A similar 
enhancement is found for SO, but only in the western lobe. This contrasts the abundances measured in IC443, where those of both CS and SO were found to be quite similar to those in TMC-1. Unfortunately, upper limits placed on the SiO abundance in L1551 are not significant.

Barnard 1 is another dark cloud which shows evidence of an outflow associated with a young low mass star. Recent studies of this object by Bachiller, Menten, and del Río-Alvarez (1990) show several clumps in CS that contain low-luminosity young stellar objects detected by IRAS. The clumps exhibit $T_{k} \geq 12 K$. Toward one such object, IRAS03301+3057, high velocity wings with a dispersion up to $40 \mathrm{~km} / \mathrm{s}$ have been observed on $\mathrm{J}=2 \rightarrow 1$ spectra of CO. Similar, less pronounced wings were also detected on $\mathrm{SO}$ and $\mathrm{SiO}$ spectra (see Figure 2). The wings are thought to arise from a high velocity flow originating with mass loss from IRAS03301+3057. The gas tracing the outflow is therefore likely to be shocked.

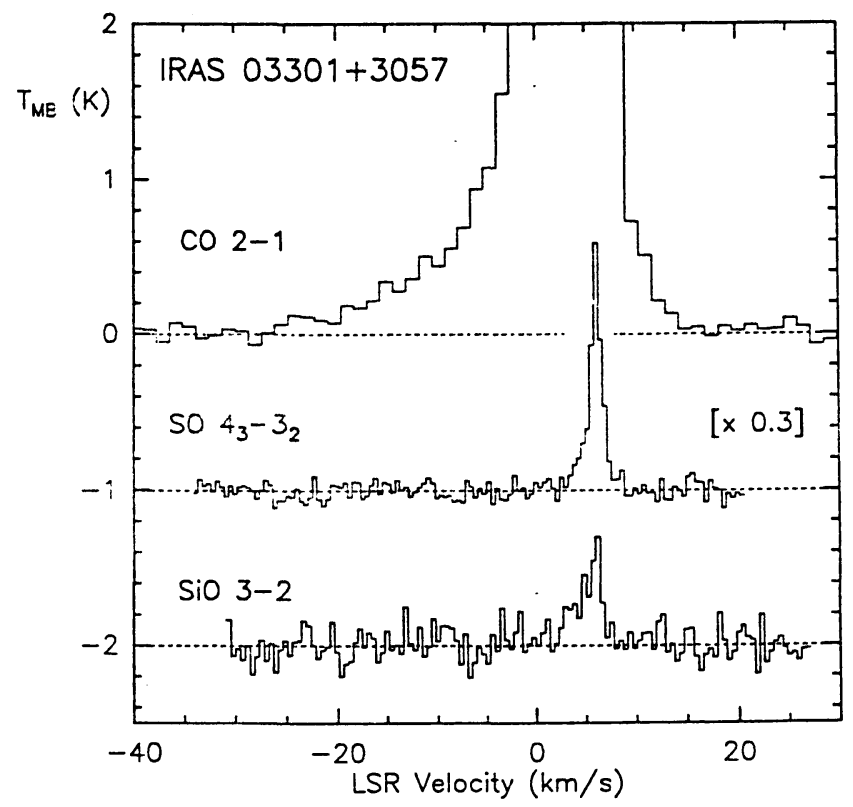

Figure 2. CO, SO, and SiO spectra observed towards B1 (from Bachiller et al. 1990).

Bachiller et al. measured molecule abundances towards the outflow. Unfortunately, they did not observe $\mathrm{HCN}$, so their abundances are referenced to $\mathrm{H}_{2}$. Curiously, their CS and SO concentrations are similar to TMC-1. SiO, on the other hand, is significantly enriched. The abundance of $\mathrm{HCO}^{+}$is slightly higher in $\mathrm{B} 1$ than $\mathrm{TMC}-1$, but those of $\mathrm{HC}_{3} \mathrm{~N}$ and $\mathrm{HC}_{5} \mathrm{~N}$ are significantly lower. 


\section{Conclusions}

The chemical affects of shocks in the regions examined here do vary. There appears to be a trend towards enhanced abundances of certain sulfur-bearing species such as $\mathrm{SO}, \mathrm{SO}_{2}, \mathrm{H}_{2} \mathrm{~S}$, and, in some cases, CS. However, as was found for IC443, this may not always be the case. $\mathrm{HCO}^{+}$ tends to show a small abundance enhancement in most of the shocked sources examined here, but additional studies need to be performed before this is a certainty. Curiously, certain large organic species show evidence of shock enhancement, in particular ethyl cyanide, while others prefer more quiescent gas such as dimethyl ether and methyl acetylene. This is difficult to explain. One species, however, seems to always be present in hot, shocked gas: $\mathrm{SiO}$. Why $\mathrm{SiO}$ is abundant in shocked regions may be due to high temperature reactions (Langer and Glassgold 1990), and/or grain destruction. Clearly, additional studies need to be carried out before the chemical effects of a shock wave can be satisfactorily evaluated.

\section{References}

Bachiller, R., Menten, K. M., and del Río-Alvarez , S. (1990), Astr. Ap., 236, 461.

Blake, G. A., Sutton, E. C., Masson, C. R., and Phillips, T. G. (1987), Ap. J. 315, 621.

DeNoyer, L. K. (1979a), Ap. J. (Letters), 232, L165.

DeNoyer, L. K. (1983), Ap. J., 264, 141.

DeNoyer, L. K. and Frerking, M. A. (1981), Ap. J. (Letters), 246, L37.

Dickman, R. L., Snell, R. L., Ziurys, L. M., and Huang, Y.-L. (1991), Ap. J., submitted.

Jewell, P. R., Hollis, J. M., Lovas, F. J., and Synder, L. E. (1989), Ap. J. S., 므, 833.

Johansson, L. E. B. et al. (1984), Astr. Ap., 130, 227.

Langer, W. D. and Glassgold, A. E. (1990), Ap. J., 352, 123.

Masson, C. R., et al. (1985), Ap. J. (Letters), 295, L47.

Minh, Y. C., Ziurys, L. M., Irvine, W. M., and McGonagle, D. (1990), Ap. J., 360, 136.

McKee, C. F., Chernhoff, D., and Hollenbach, D. (1984), Galactic and Extragalactic Infrared

Spectroscopy, ed. M. Kessler and J. Phillips (Dordrecht: Reidel) p. 103.

Mitchell, G. F. (1984), Ap. J., 287, 665.

Neufeld, D. A. and Dalgarno, A. (1989), Ap. J. 340, 869.

Plambeck, R. L., Vogel, S. N., Wright, M. C. H., Bieging, J. H., and Welch, W. J. (1985),

Symposium on MM and sub MM Astronomy, ed. J. Gomez-Gonzales (URSI), p. 235.

Plambeck, R. L. and Snell, R. L. (1991), Ap. J., submitted.

Schloerb, F. P. and Snell, R. L. (1980), Ap. J., 295, 490.

Snell, R. L., Loren, R. B., and Plambeck, R. L. (1980), Ap. J. (Letters), 239, L17.

Sutton, E. C., Blake, G. A. Masson, C. R., and Phillips, T. G. (1985), Ap. J. S., 58, 341.

Wootten, H. A. (1978), Ph.D. Thesis, University of Texas at Austin.

Wootten, H. A. (1981), Ap. J. 245, 105.

Wynn-Williams, C. G., Genzel, R., Becklin, E. E., and Downes, D. (1984), Ap. J. 281, 172.

Ziurys, L. M., Snell, R. L., and Dickman, R. L. (1989), Ap. J., 341, 857.

Ziurys, L. M., Friberg, P., and Irvine, W. M. (1989), Ap. J., 343, 201.

Ziurys, L. M. (1990), Evolution of the Interstellar Medium, ed. L. Blitz (San Francisco:

A.S.P.), p. 229.

Ziurys, L. M., and McGonagle, D. (1991), Ap. J. S., submitted.

Ziurys, L. M., Wilson, T. L., Waterlout, J. (1991), in preparation. 


\section{QUESTIONS AND ANSWERS}

B.E.Turner: Si species, although observed to be enhanced in shocked regions, may simply represent the evaporation of refractory ice mantles from grains, which occours at $\sim 90 \mathrm{~K}$. We find SiO abundances in IC 443G to be 100 to 1000 times lower than predicted by either non-disssociative or dissociative models. Further, line widths of SiO in most sources are not unusual, but would be expected to be wider if involved in the shocks. There is no direct evidence that the Si-species themselves are "hot" in the sense of large rotational temperatures, as would be expected if shock produced. One only needs a small fraction of refractory ice mantles to be evaporated, even in Orion(KL), to produce the observed SiO abundances (cf. Turner 1991, Ap.J. in press). In grain also, only a small fraction of total Si is in SiO, contrary to shock models.

L.M.Ziurys: Ziurys and Friberg back in 1987 (Ap.J.(Letters) 314,L49) noted that only a small fraction of silicon was needed to account for all gas phase silicon molecules in Orion. It could well be that enhancement of $\mathrm{Si}$ compounds in shocked regions may result from grain evaporation. However, SiO does exhibit high velocity wings quite often (i.e., see Dounes et al. 1982,Ap.J.(Lett),252,L29). In fact, it was the presence of an asymmetric wing in $\mathrm{SiO}$ that indicated the existence of the outflow at Orion-S (Ziurys and Friberg 1987). Asymmetric wings are also seen in SiO spectra towards BI (Bachilller et al.(1990),Astr.Ap. 236, 461). Also there are various indications that $\mathrm{SiO}$ in outflows is hot. Towards Orion, the brightness temperature in SiO is near $230 \mathrm{~K}$ ! This is clealy hot! Also, LVG modeling of the $J=2 \rightarrow 1 / J=5 \rightarrow 4 \mathrm{SiO}$ transitions towards the Orion-S outflow indicates a kinetic temperature near $100 \mathrm{~K}$ (Ziurys et al. Ap.J.(Lett),356,L25,1990).

Y.Wu: Some molecular species are indeed enhanced in shock region, it can include CS; but CS is also overabundant in dense cores without source, where there is no shock caused by stellar wind. Have you any idea about this?

L.M.Ziurys: It is not clear yet whether CS is actually enhanced in shocked gas. It could appear to be overabundant in dense cores due to some low temperature reaction that hasn't been properly included in chemical models. About $50 \%$ of reaction rates used in theoretical models have never been measured experimentally at room temperature, let alone at $T \sim 10 \mathrm{~K}$.

M.Guelin: There is another category of sources where we have a clear evidence of shock chemistry: the envelopes of some evolved stars (e.g. protoplanetary nebulae). There is a high velocity $v_{\text {eap }}>200 \mathrm{~km} / \mathrm{s}$ outflow in CRL 618 where half a dozen of molecules have been detected (CO, $\mathrm{HCN}, \mathrm{HC}_{3} \mathrm{~N}, \mathrm{HCO}^{+}, \mathrm{CN}$ ).

L.M.Zuirys: CRL 618 would be another interesting source to examine chemical abundances in.

S.P.Tarafdar: Dr.Ohishi showed yesterday that $\mathrm{HC}_{3} \mathrm{~N}$ and $N H_{3}$ regions are different whereas you have put both species originating in the hot core of Orion-KL. Is it due to difference in resolution or $\mathrm{HC}_{8} \mathrm{~N}$ and $\mathrm{NH}_{\mathrm{g}}$ in Orion-KL do originate from the same region?

L.M.Zuirys: Both $\mathrm{HC}_{3} \mathrm{~N}$ and $\mathrm{NH}_{8}$ are present in the Orion hot core, plateau, and ridge components, examining single dish spectra. However, their detailed distributions do seem to differ, if one looks at VLA $\mathrm{NH}_{3}$ maps vs Hat Creek $\mathrm{HC}_{3} \mathrm{~N}$ maps. Assigning species to various Orion components is somewhat of a generalization. 